\title{
UCRL-TR-209603
}

LAWRENCE LIVERMORE N A TION A L LABORATORY
Low Voltage High Precision Spatial Light ModulatorsFinal Report

Alexandros P. Papavasiliou

February 10, 2005 
This document was prepared as an account of work sponsored by an agency of the United States Government. Neither the United States Government nor the University of California nor any of their employees, makes any warranty, express or implied, or assumes any legal liability or responsibility for the accuracy, completeness, or usefulness of any information, apparatus, product, or process disclosed, or represents that its use would not infringe privately owned rights. Reference herein to any specific commercial product, process, or service by trade name, trademark, manufacturer, or otherwise, does not necessarily constitute or imply its endorsement, recommendation, or favoring by the United States Government or the University of California. The views and opinions of authors expressed herein do not necessarily state or reflect those of the United States Government or the University of California, and shall not be used for advertising or product endorsement purposes.

This work was performed under the auspices of the U.S. Department of Energy by University of California, Lawrence Livermore National Laboratory under Contract W-7405-Eng-48. 


\title{
FY04 LDRD Final Report \\ Low-Voltage High-Precision Spatial Light Modulator LDRD Project Tracking Code: 04-ERD-014 Alexandros Papavasiliou, Principal Investigator
}

\begin{abstract}
The goal of this project was to make LLNL a leader in Spatial Light Modulators (SLMs) by developing the technology that will be needed by the next generation of SLMs. We would use new lower voltage actuators and bond those actuators directly to controlling circuitry to break the fundamental limitations that constrain current SLM technology.
\end{abstract}

This three-year project was underfunded in the first year and not funded in the second year. With the funding that was available, we produced actuators and designs for the controlling circuitry that would have been integrated in the second year.

Spatial light modulators (SLMs) are arrays of tiny movable mirrors that modulate the wave-fronts of light. SLMs can correct aberrations in incoming light for adaptive optics or modulate light for beam control, optical communication and particle manipulation. MicroElectroMechanical Systems (MEMS) is a technology that utilizes the microfabrication tools developed by the semiconductor industry to fabricate a wide variety of tiny machines. The first generation of MEMS SLMs have improved the functionality of SLMs while drastically reducing per pixel cost making arrays on the order of 1000 pixels readily available. These MEMS SLMs however are limited by the nature of their designs to be very difficult to scale above 1000 pixels and have very limited positioning accuracy. By co-locating the MEMS mirrors with CMOS electronics, we will increase the scalability and positioning accuracy. To do this we will have to make substantial advances in SLM actuator design, and fabrication.

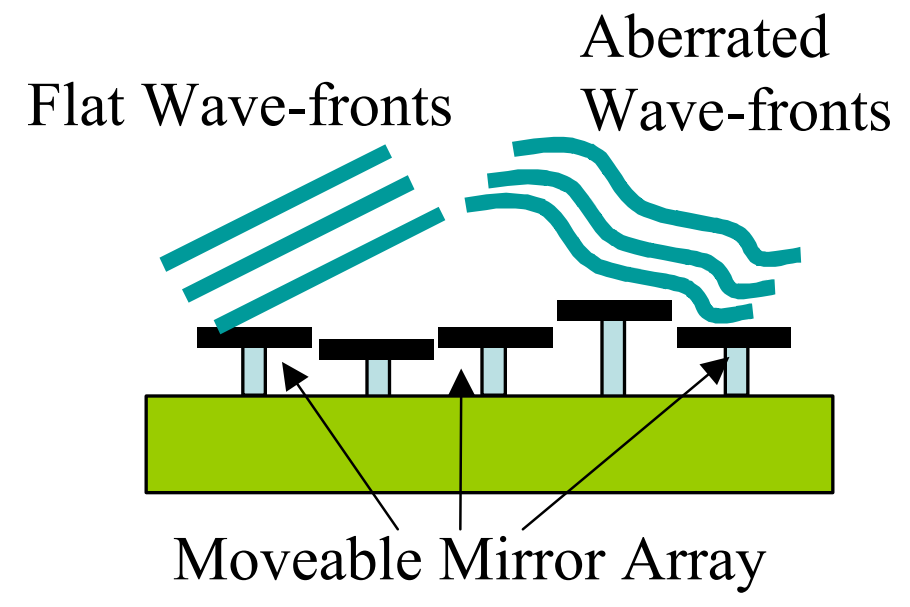

Figure 1. A cartoon of a Spatial Light Modulator modulating the wave fronts of incident light. 


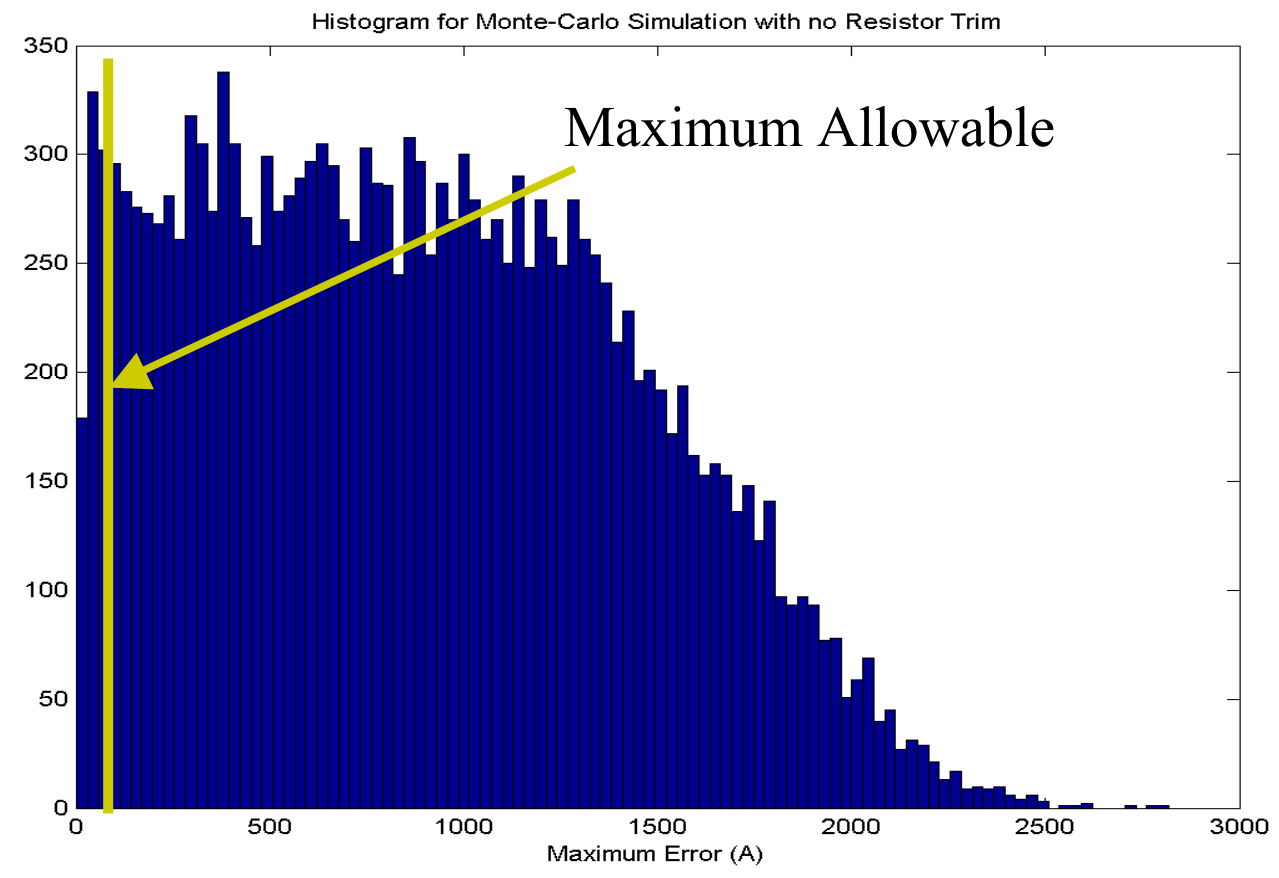

Figure 2. A histogram of the errors expected from a SLM actuator with open-loop control compared to the maximum allowable error of the DARPA CCIT project. The data was produced by a Monte-Carlo simulation of SLM behavior based on typical MEMS processing tolerances.

\section{Project Goals}

The ultimate goal of this project is to make LLNL a leader in spatial light modulator technology. We would do that by demonstrating a small array (19 pixels) of MEMS mirrors that have an integrated closed-loop control system and a clear path to scaling that system up to thousands of pixels. As a part of that we will learn about bonding MEMS structures directly to controlling electronics. This technology would be valuable not only to spatial light modulator work but to many other projects at the laboratory.

\section{Relevance to Lab Mission}

This work is relevant to a number of laboratory missions. These SLMs can be used by astronomy projects such as the LSST and the TMT. The technology developed by this project will also be useful for the nanolaminate deformable mirror work being carried out by LLNL. In biology, SLMs are useful for imaging and for particle manipulation. SLMs are used for laser control and beam steering; there is a potential place in the NIF preamplifiers.

The bonding of MEMS to electronics has wide ranging applications such as large arrays chemical and biological sensors. Electronics integrated with other devices could minimize package size and complexity in small autonomous devices. 


\section{Methods and Results}

This project has two major components which were to be integrated together: An effort to produce actuators that could carry mirrors and be integrated with electronics, and an effort do create the electronics that would control those actuators.

\section{Actuators:}

We created first-principles models of actuator structure, created optimal designs based on modeling work, designed a layout of the $1^{\text {st }}$ generation MEMS structures, fabricated the $1^{\text {st }}$ generation MEMS structures, demonstrated un-bonded MEMS structures and fabricated and delivered MEMS and dummy wafers to bonding sub-contractor. Those devices were bonded to dummy chips by the sub contractor but by the end of the year we had not succeeded in releasing bonded chips from their handle wafers.

Electronics:

We have moved towards a layout for the electronics chip which will eventually be bonded to the MEMS and control the SLM pixels. We have a schematic design for the analog circuits. Getting the software, and technology files necessary to turn our schematic design into a layout, and getting NDAs in place so that we will have permission to use them, has been exceedingly difficult. By the end of the fiscal year, we had all the necessary paperwork in position and we the process of designing the control circuits for the first device had begun.

\section{Actuator Design}

The three electrode architecture:

The three-electrode actuators that were designed for this project were built upon the basic technology developed during an FY02 LDRD Feasibility study on three-electrode actuators. The feasibility study had shown that such actuators could be built and had a performance advantage over conventional two-electrode actuators. Those actuators were, however stand-alone test devices that could not be attached to mirrors or to controlling electronics.

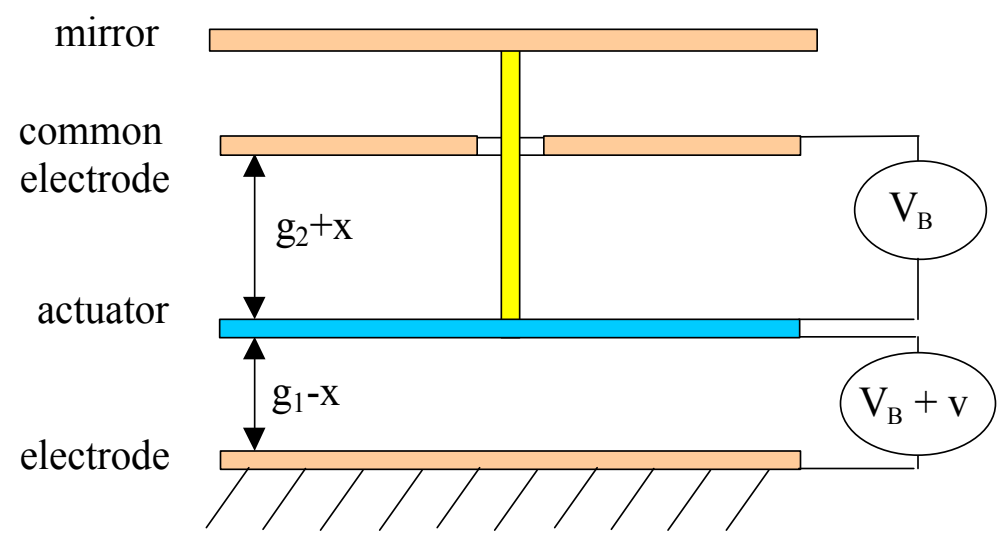

Figure3. Schematic of Three-electrode Actuator 


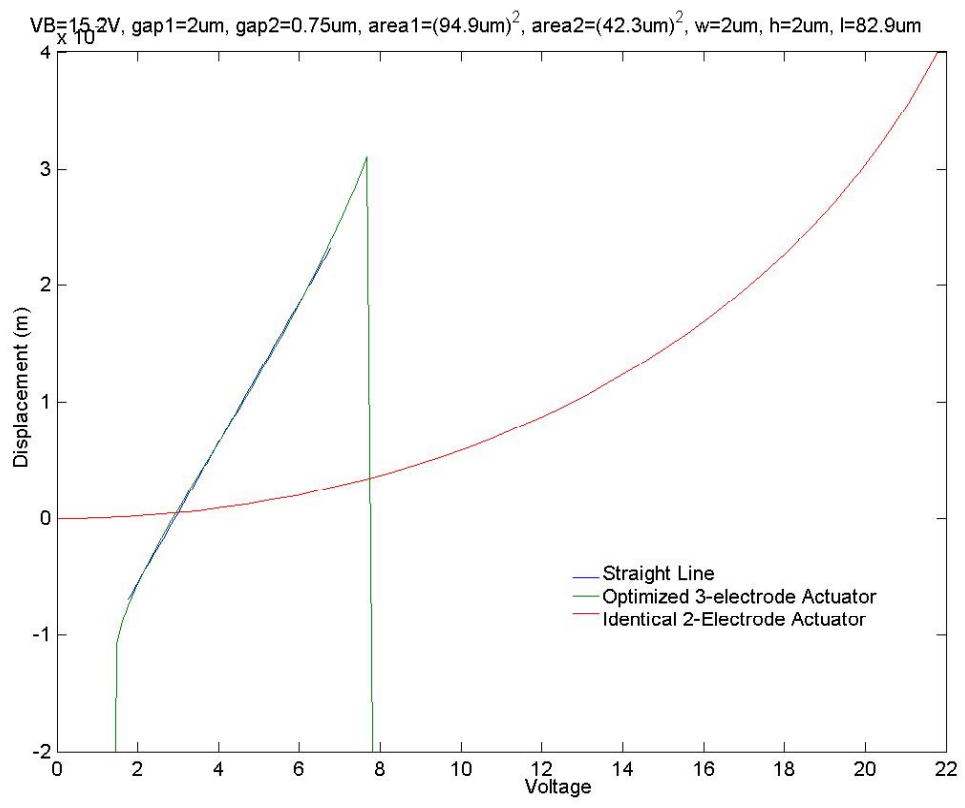

Figure 4. Typical displacement vs voltage response of three-electrode actuator as compared to a two electrode actuator with the same dimensions.

Integratable actuators

We produced actuators that could be bonded to electronics and could carry a mirror by tweaking the inexpensive MUMPS foundry process and then flipping the actuator over to be bonded to the controlling electronics. The three-electrode structure requires three separate conducting layers each of which is insulated from the other two, but connected to the electronics. In addition, the top and bottom layers must be rigidly mechanically connected but electrically isolated.

The MUMPS process is a three-layer polysilicon MEMS process. Poly0 is fabricated on top of a nitride film, polyl is fabricated on top of a sacrificial silicon dioxide layer that is fabricated on top of the poly0, and poly2 is fabricated on top of another silicon dioxide layer that is fabricated on top of poly 1 . The polysilicon layers can be connected through vias cut in the silicon dioxide layers. The polysilicon layers are "released" by etching away the silicon dioxide. We then bond the polysilicon to the electronics by flipping it over and pressing it against gold bumps. 


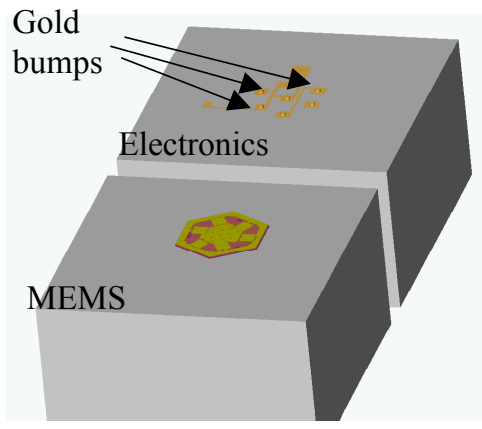

MEMS and

electronics

fabricated

separately

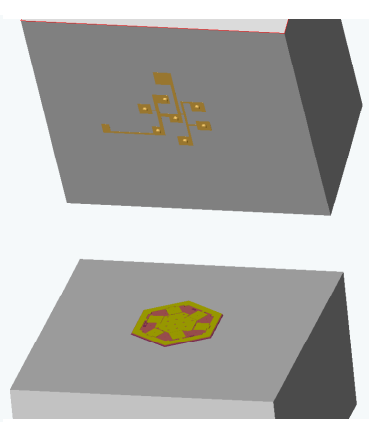

Chips flipped over

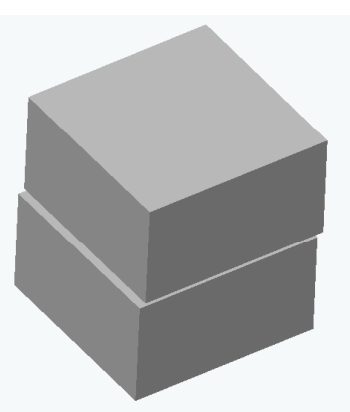

Chips bonded together
MEMS device

Electronics

MEMS handle wafer removed

Figure5 Die with gold bumps being flipped over to bond to MEMS structure.

All the connections to the substrate were made with gold bumps bonding to pads on the poly2 layer. We rigidly attached the middle electrode, made from poly1, to the substrate with vias attaching to a poly 2 bond pad. We suspended the top electrode (made from poly0) and bottom electrode made from poly2 by springs cut out of the poly1 layer. Each of these electrodes were attached by vias to the poly 1 spring, and each of the poly 1 springs was attached to a poly 2 bond pad. We mechanically connected the top electrode to the bottom electrode by a plug made by stacking up pieces of poly0, poly1, and poly 2 all connected by vias. The poly0 portion of the plug was attached through the nitride layer to the poly0 top electrode.

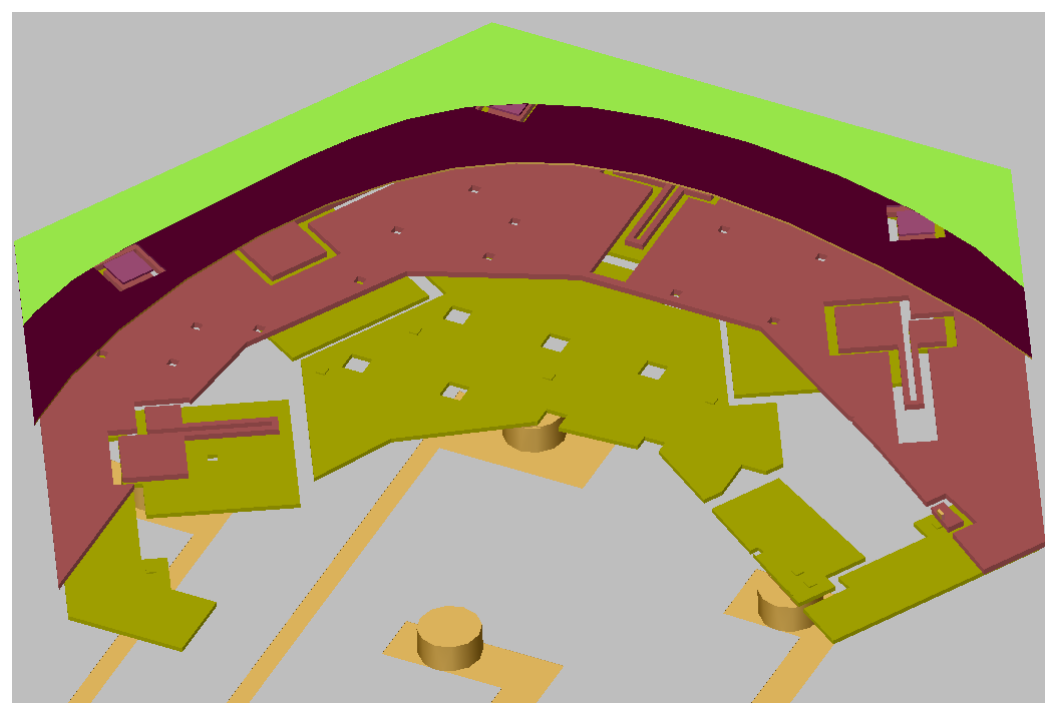

Figure 6. Cutaway drawing of the actuator showing bond pads(gold) bottom polysilicon layer Poly2(olive green) middle polysilicon layer, poly1, (red) top polysilicon layer, Poly0 (black) and nitride layer (light green). 


\section{Fabrication}

The actuators were fabricated at the MUMPS foundry. Dummy chips were produced at LLNL and gold bumps were attached to the dummy chips by the bonding subcontractor MicroAssembly Technologies.

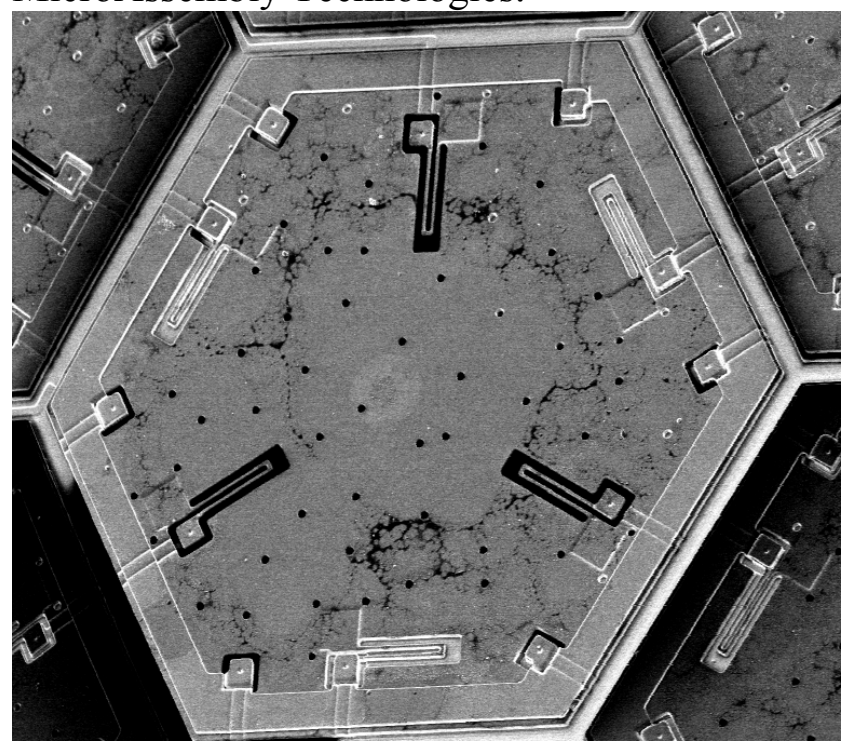

Figure7. An SEM image of the realized polysilicon structure.

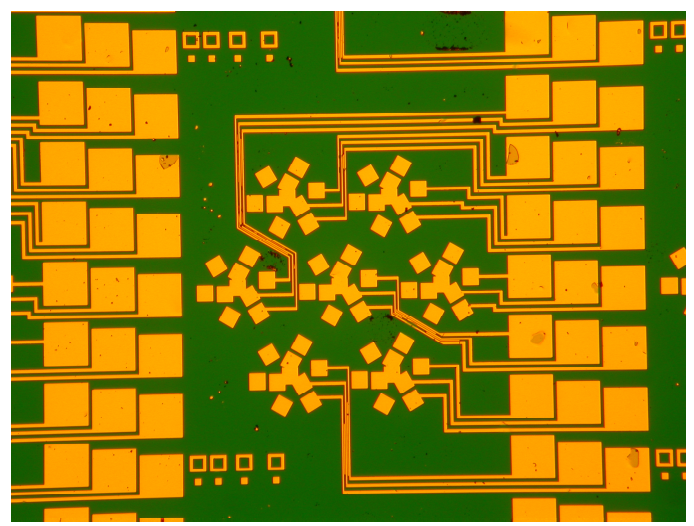

Figure 8. A photograph of the gold bond pads for an array of actuators. 


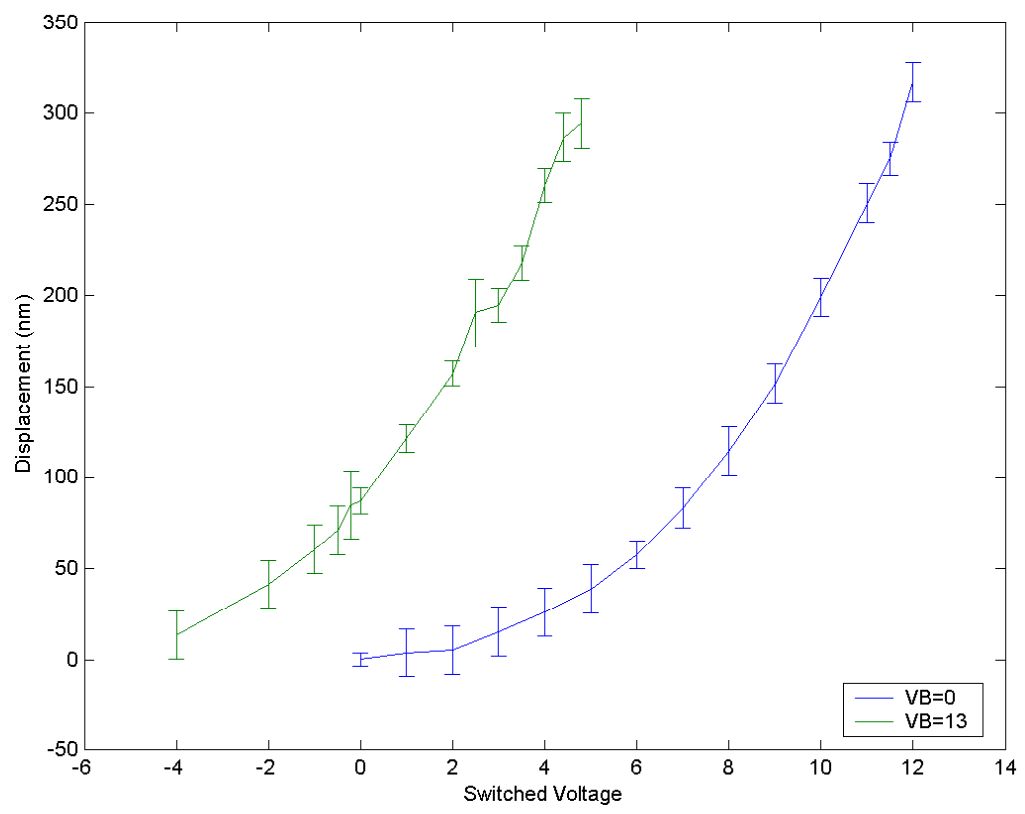

Figure 9. Displacement vs. Voltage for a test actuator.

\section{Handle wafer release:}

We did not find a suitable handle wafer release method before the funding for this project ran out. We did however attempt to remove the handle wafer two different ways.

The basic problem is that the MEMS actuator is fabricatated on top of and attached to a $525 \mu \mathrm{m}$ thick silicon wafer. The silicon wafer needs to be removed from the back side of the MEMS actuator after the actuator has been bonded to the electronics without destroying either the actuator or the electronics.

$\mathrm{KOH}$ parylene release

The first strategy we used was to etch away the handle wafer with a heated $\mathrm{KOH}$ solution and protect the mems strucutures with a coating of parylene. The etch would be stopped by the silicon nitride layer before etching away the MEMS structures. While parylene proved to be impervious to potassium hydroxide solution, it did not adhere well to the silicon. So although the parylene survived, the etchant seeped between the parylene and the silicon eventually reaching the MEMS structures and destroying them

RIE etching release

The second strategy we tried was to use a reactive ion etch (RIE) to remove the handle wafer. In this strategy we protected the MEMS structures with photoresist to prevent any horizontal etching under the dummy chip. This strategy was also not successful. While we were able to etch down to the nitride layer, non uniformities in the etch and non uniformities in the photoresist protective layer allowed the RIE etcher to remove the MEMS in some areas without removing the entire handle wafer in others. This strategy seems like it could be successful if the stop layer, the silicon nitride in this case, were substantially thicker and if more care was put into the photoresist deposition. However, the project came to an end before these strategies could be tried. 


\section{Conclusion.}

This project succeeded in designing a three-electrode type actuator which could be used to with a mirror and can be bonded directly to electronics. Those actuators were fabricated and test structures were tested. Some preliminarily bonding experiments were carried out but MEMS were not successfully released before the project ended. 